\title{
Overdetermined problems in unbounded domains with Lipschitz singularities
}

\section{Alberto Farina and Enrico Valdinoci}

Abstract
We study the overdetermined problem

$$
\left\{\begin{array}{cc}
\Delta u+f(u)=0 & \text { in } \Omega, \\
u=0 & \text { on } \partial \Omega, \\
\partial_{\nu} u=c & \text { on } \Gamma,
\end{array}\right.
$$

where $\Omega$ is a locally Lipschitz epigraph, that is $C^{3}$ on $\Gamma \subseteq \partial \Omega$, with $\partial \Omega \backslash \Gamma$ consisting in nonaccumulating, countably many points.

We provide a geometric inequality that allows us to deduce geometric properties of the sets $\Omega$ for which monotone solutions exist.

In particular, if $\mathscr{C} \in \mathbb{R}^{n}$ is a cone and either $n=2$ or $n=3$ and $f \geqslant 0$, then there exists no solution of

$$
\left\{\begin{array}{cc}
\Delta u+f(u)=0 & \text { in } \mathscr{C}, \\
u>0 & \text { in } \mathscr{C}, \\
u=0 & \text { on } \partial \mathscr{C}, \\
\partial_{\nu} u=c & \text { on } \partial \mathscr{C} \backslash\{0\} .
\end{array}\right.
$$

This answers a question raised by Juan Luis Vázquez.

\section{Introduction}

Let $n \geqslant 2$. We consider an epigraph in $\mathbb{R}^{n}$, that is

$$
\Omega:=\left\{\left(x^{\prime}, x_{n}\right) \in \mathbb{R}^{n-1} \times \mathbb{R} \text { s.t. } x_{n}>\Phi\left(x^{\prime}\right)\right\} .
$$

We suppose that $\Omega$ is locally Lipschitz and that it is $C^{3}$ except, at most, at a countable family of points that do not accumulate.

2000 Mathematics Subject Classification: 35J25, 35J20, 35B65.

Keywords: Elliptic partial differential equations, rigidity results, nonexistence of solutions. 
Explicitly, we suppose that there exists $\mathscr{J} \subseteq \mathbb{N}$ and a family

$$
\mathscr{F}:=\left\{p_{j}^{\prime} \in \mathbb{R}^{n-1}, j \in \mathscr{J}\right\}
$$

such that

$$
\inf _{j, k \in \mathscr{J}}\left|p_{j}^{\prime}-p_{k}^{\prime}\right|>0
$$

and

$$
\Phi \in C^{3}\left(\mathbb{R}^{n-1} \backslash\left(\bigcup_{j \in \mathscr{J}} p_{j}^{\prime}\right)\right) .
$$

Notice that $\nabla \Phi$ exists a.e.: we suppose that

$$
\|\nabla \Phi\|_{L^{\infty}(K)}<+\infty
$$

for any bounded set $K$ in $\mathbb{R}^{n-1}$.

We denote $p_{j}:=\left(p_{j}^{\prime}, \Phi\left(p_{j}^{\prime}\right)\right)$ and

$$
\Gamma:=\partial \Omega \backslash\left(\bigcup_{j \in \mathscr{J}} p_{j}\right) .
$$

We remark that, by construction, the exterior derivative $\nu$ is always well defined at points of $\Gamma$.

Given $c \in \mathbb{R}$, we will study the following overdetermined elliptic problem:

$$
\left\{\begin{aligned}
\Delta u+f(u)=0 & & \text { in } \Omega, \\
u=0 & & \text { on } \partial \Omega, \\
\partial_{\nu} u=c & & \text { on } \Gamma .
\end{aligned}\right.
$$

We will prove a geometric inequality for solutions of (0.2) and some rigidity results in low dimension.

For this, we introduce some notation.

Given a smooth function $v$, one may consider the level sets of $v$ : in the vicinity of $\{\nabla v \neq 0\}$, these level sets are smooth manifolds, so one can consider the principal curvatures

$$
\kappa_{1}, \ldots, \kappa_{n-1}
$$

at any point of such manifolds.

We set

$$
\mathscr{K}:=\sqrt{\kappa_{1}^{2}+\cdots+\kappa_{n-1}^{2}} .
$$

Also, it is customary to consider the tangential gradient along level sets of $v$ at these points, that is

$$
\nabla_{T} g:=\nabla g-\left(\nabla g \cdot \frac{\nabla v}{|\nabla v|}\right) \frac{\nabla v}{|\nabla v|} .
$$


Thus, we may state the main results of this paper as follows:

Theorem 1. Let $u \in C^{2}(\Omega \cup \Gamma) \cap C(\bar{\Omega}) \cap W_{\text {loc }}^{1, \infty}(\Omega)$ be a solution of $(0.2)$, with $\partial_{n} u>0$ in $\Omega$.

Then, for any $\xi \in C_{0}^{\infty}\left(\mathbb{R}^{n}\right)$,

$$
\int_{\Omega}\left(|\nabla u|^{2} \mathscr{K}^{2}+\left|\nabla_{T}\right| \nabla u||^{2}\right) \xi^{2} \leqslant \int_{\Omega}|\nabla u|^{2}|\nabla \xi|^{2} .
$$

Theorem 2. Let $u \in C^{2}(\Omega \cup \Gamma) \cap W^{1, \infty}(\Omega)$ be a solution of $(0.2)$, with $u>0$ in $\Omega$ and $\Omega$ globally Lipschitz.

Suppose

- either that $n=2$

- or that $n=3$ and $f \geqslant 0$.

Then, $\Omega$ cannot be coercive, that is, it cannot be that

$$
\lim _{\left|x^{\prime}\right| \rightarrow+\infty} \Phi\left(x^{\prime}\right)=+\infty .
$$

The result in Theorem 1 may be seen as a weighted Poincaré inequality. Similar inequalities have been used first in $[5,6]$, where no boundary term was present, and in $[2,3]$ to deduce symmetry results for PDEs. In [4] related inequalities have been used for problems like (0.2) in smooth domains. Differently than [4], in this paper we take into account also domains with Lipschitz singularities: indeed, when the domains are smooth, Theorems 1 and 2 here boil down to Theorems 1.1 and 1.6 in [4].

As a side remark, we also notice that the left hand side of $(0.3)$ is welldefined, since $\nabla u \neq 0$ in $\Omega$.

We now use Theorem 2 in order to answer a question posed to us by Juan Luis Vázquez [7]. For this, let $\mathscr{C}$ be a cone.

More precisely, if $n=2$, we write

$$
t^{+}:=\left\{\begin{array}{cl}
|t| & \text { if } t>0, \\
0 & \text { if } t \leqslant 0,
\end{array} \quad \text { and } \quad t^{-}:=\left\{\begin{array}{cl}
|t| & \text { if } t<0 \\
0 & \text { if } t \geqslant 0
\end{array}\right.\right.
$$

and, given $\alpha^{+}, \alpha^{-} \in(0,+\infty)$, we define the cone

$$
\mathscr{C}:=\left\{\left(x_{1}, x_{2}\right) \in \mathbb{R}^{2} \text { s.t. } x_{2}>\alpha^{+} x_{1}^{+}+\alpha^{-} x_{1}^{-}\right\} .
$$

When $n \geqslant 3$, given $\alpha \in(0,+\infty)$, we write the cone as

$$
\mathscr{C}:=\left\{\left(x^{\prime}, x_{n}\right) \in \mathbb{R}^{n-1} \times \mathbb{R} \text { s.t. } x_{n}>\alpha|x|\right\} .
$$


With this notation, we obtain the following result:

\section{Corollary 3. If}

- either $n=2$

- or $n=3$ and $f \geqslant 0$,

then there exists no solution $u \in C^{2}(\overline{\mathscr{C}} \backslash\{0\}) \cap W^{1, \infty}(\mathscr{C})$ of

$$
\left\{\begin{array}{rlrl}
\Delta u+f(u)=0 & & \text { in } \mathscr{C} \\
u>0 & & \text { in } \mathscr{C} \\
u=0 & & \text { on } \partial \mathscr{C} \\
\partial_{\nu} u & =c & & \text { on } \partial \mathscr{C} \backslash\{0\}
\end{array}\right.
$$

Corollary 3 is a simple consequence of Theorem 2. We also recall that solutions of (0.4) satisfy

$$
\partial_{n} u(x)>0 \text { for any } x \in \Omega,
$$

thanks to Theorem 1.3 in [1].

We prove Theorems 1 and 2 in the forthcoming Sections 1 and 2, respectively.

\section{Proof of Theorem 1}

Let now $\xi \in C_{0}^{\infty}\left(\mathbb{R}^{n}\right)$.

We define

$$
\rho:=\inf _{j, k \in \mathscr{J}}\left|p_{j}^{\prime}-p_{k}^{\prime}\right| .
$$

We recall that $\rho>0$ because of (0.1).

We fix $K>2$ and $\eta>0$ such that

$$
\eta \leqslant \min \left\{1, \frac{\rho}{2}, \frac{1}{\log K}\right\} .
$$

We define

$$
\tau_{\eta, K}(x):=\left\{\begin{array}{cl}
0 & \text { if }|x| \leqslant \eta / K, \\
\frac{\log |x|-\log (\eta / K)}{\log K} & \text { if } \eta / K<|x| \leqslant \eta, \\
1 & \text { if }|x|>\eta .
\end{array}\right.
$$


Notice that $\tau_{\eta, K}$ is Lipschitz continuous and

$$
\left|\nabla \tau_{\eta, K}(x)\right| \leqslant \frac{\chi_{\left(B_{\eta} \backslash B_{\eta / K}\right)}(x)}{|x| \log K} .
$$

Here above, as customary, we have denoted by $\chi_{A}$ the characteristic function of the set $A$.

We also set

$$
\xi_{\eta, K}(x):=\xi(x) \cdot \prod_{j \in \mathscr{J}} \tau_{\eta, K}\left(x-p_{j}\right) .
$$

This function is well-defined, since $\tau_{\eta, K}\left(x-p_{j}\right)=1$ for $x \notin B_{\eta}\left(p_{j}\right)$ and these balls are disjoint.

We now take $\Omega_{\eta, K}$ to be an open set with $C^{3}$ boundary such that

$$
\Omega \backslash\left(\bigcup_{j \in \mathscr{J}} B_{\eta /(2 K)}\left(p_{j}\right)\right) \subset \Omega_{\eta, K} \subset \Omega \backslash\left(\bigcup_{j \in \mathscr{J}} B_{\eta /(4 K)}\left(p_{j}\right)\right) .
$$

We make use of (1.4) of [4] to obtain that

$$
\begin{aligned}
& \int_{\Omega_{\eta, K}} \quad\left(|\nabla u|^{2} \mathscr{K}^{2}+\left|\nabla_{T}\right| \nabla u||^{2}\right) \varphi^{2} \\
& \quad+\limsup _{\epsilon \rightarrow 0^{+}} \int_{\partial \Omega_{\eta, K}} \frac{\varphi^{2}}{\epsilon+\partial_{n} u}\left(|\nabla u|^{2} \partial_{n, \nu}^{2} u-\partial_{i, \nu}^{2} u \partial_{i} u \partial_{n} u\right) \leqslant \\
& \quad \leqslant \int_{\Omega_{\eta, K}}|\nabla u|^{2}|\nabla \varphi|^{2}
\end{aligned}
$$

for any $\varphi \in W_{0}^{1, \infty}\left(\mathbb{R}^{n}\right)$.

On the other hand, from (3.15) in [4] and (0.2) here, we know that

$$
|\nabla u|^{2} \partial_{n, \nu}^{2} u-\partial_{i, \nu}^{2} u \partial_{i} u \partial_{n} u=0 \quad \text { on } \Gamma \text {. }
$$

Also, by construction,

$$
\xi_{\eta, K}=0 \quad \text { in } \bigcup_{j \in \mathscr{J}} B_{\eta /(2 K)}\left(p_{j}\right) .
$$

From (1.4) and (1.5) we thus obtain

$$
\begin{aligned}
\int_{\partial \Omega_{\eta, K}} & \frac{\xi_{\eta, K}^{2}}{\epsilon+\partial_{n} u}\left(|\nabla u|^{2} \partial_{n, \nu}^{2} u-\partial_{i, \nu}^{2} u \partial_{i} u \partial_{n} u\right)= \\
= & \sum_{j \in \mathscr{J}} \int_{\partial \Omega_{\eta, K} \cap B_{\eta /(2 K)}\left(p_{j}\right)} \frac{\xi_{\eta, K}^{2}}{\epsilon+\partial_{n} u}\left(|\nabla u|^{2} \partial_{n, \nu}^{2} u-\partial_{i, \nu}^{2} u \partial_{i} u \partial_{n} u\right)=0 .
\end{aligned}
$$


Consequently, by taking $\varphi:=\xi_{\eta, K}$ in (1.3), we obtain

$$
\int_{\Omega_{\eta, K}}\left(|\nabla u|^{2} \mathscr{K}^{2}+\left|\nabla_{T}\right| \nabla u||^{2}\right) \xi_{\eta, K}^{2} \leqslant \int_{\Omega_{\eta, K}}|\nabla u|^{2}\left|\nabla \xi_{\eta, K}\right|^{2} .
$$

Also, recalling (1.1) and (1.2), a straightforward computation gives that

$$
\begin{aligned}
& \left|\nabla \xi_{\eta, K}(x)\right|-|\nabla \xi(x)| \leqslant\left|\nabla \xi_{\eta, K}(x)-\nabla \xi(x)\right| \leqslant \\
& \leqslant\|\xi\|_{W^{1, \infty}\left(\mathbb{R}^{n}\right)} \sum_{j \in \mathscr{J}}\left(1+\frac{1}{\left|x-p_{j}\right| \log K}\right) \chi_{\left(B_{\eta}\left(p_{j}\right) \backslash B_{\eta / K}\left(p_{j}\right)\right)}(x) \\
& \quad \leqslant 2\|\xi\|_{W^{1, \infty}\left(\mathbb{R}^{n}\right)} \sum_{j \in \mathscr{J}} \frac{\chi_{\left(B_{\eta}\left(p_{j}\right) \backslash B_{\eta / K}\left(p_{j}\right)\right)}(x)}{\left|x-p_{j}\right| \log K} .
\end{aligned}
$$

We now fix an auxiliary parameter $\delta>0$ and we use a scaled Cauchy Inequality to deduce from (1.7) that

$$
\begin{aligned}
\left|\nabla \xi_{\eta, K}(x)\right|^{2} \leqslant(1 & +\delta)|\nabla \xi(x)|^{2} \\
& +C_{\delta}\|\xi\|_{W^{1, \infty}\left(\mathbb{R}^{n}\right)}^{2}\left[\sum_{j \in \mathscr{J}} \frac{\chi_{\left(B_{\eta}\left(p_{j}\right) \backslash B_{\eta / K}\left(p_{j}\right)\right)}(x)}{\left|x-p_{j}\right| \log K}\right]^{2} .
\end{aligned}
$$

Since the balls $B_{\eta}\left(p_{j}\right)$ are disjoint, we can write the above inequality as

$$
\begin{aligned}
\left|\nabla \xi_{\eta, K}(x)\right|^{2} \leqslant(1 & +\delta)|\nabla \xi(x)|^{2} \\
& +C_{\delta}\|\xi\|_{W^{1, \infty}\left(\mathbb{R}^{n}\right)}^{2} \sum_{j \in \mathscr{J}} \frac{\chi_{\left(B_{\eta}\left(p_{j}\right) \backslash B_{\eta / K}\left(p_{j}\right)\right)}(x)}{\left|x-p_{j}\right|^{2}(\log K)^{2}} .
\end{aligned}
$$

Now, we denote by $\mathscr{S} \subset \mathbb{R}^{n}$ the support of $\xi$, and we define

$$
\mathscr{J}_{\mathscr{S}}:=\left\{j \in \mathscr{J} \text { s.t. } B_{\eta}\left(p_{j}\right) \cap \mathscr{S} \neq \emptyset\right\} .
$$

We remark that $\mathscr{J}_{\mathscr{S}}$ is a finite set, so we denote by $C_{\mathscr{S}} \in \mathbb{N}$ its cardinality. 
Then, by (1.8),

$$
\begin{aligned}
& \int_{\Omega_{\eta, K}}|\nabla u(x)|^{2}\left|\nabla \xi_{\eta, K}(x)\right|^{2} d x \leqslant \\
& \leqslant \int_{\mathscr{S}}|\nabla u(x)|^{2}\left[(1+\delta)|\nabla \xi(x)|^{2}+C_{\delta}\|\xi\|_{W^{1, \infty}\left(\mathbb{R}^{n}\right)}^{2} \sum_{j \in \mathscr{J}} \frac{\chi_{\left(B_{\eta}\left(p_{j}\right) \backslash B_{\eta / K}\left(p_{j}\right)\right)}(x)}{\left|x-p_{j}\right|^{2}(\log K)^{2}}\right] d x \\
& \leqslant(1+\delta) \int_{\Omega}|\nabla u(x)|^{2}|\nabla \xi(x)|^{2} d x \\
& \quad+C_{\delta}\|\xi\|_{W^{1, \infty}\left(\mathbb{R}^{n}\right)}^{2}\|u\|_{W^{1, \infty}(\mathscr{S})}^{2} \sum_{j \in \mathscr{J}_{\mathscr{S}}} \int_{\left(B_{\eta}\left(p_{j}\right) \backslash B_{\eta / K}\left(p_{j}\right)\right)} \frac{1}{\left|x-p_{j}\right|^{2}(\log K)^{2}} d x \\
& =(1+\delta) \int_{\Omega}|\nabla u(x)|^{2}|\nabla \xi(x)|^{2} d x \\
& \quad+\frac{C_{\delta}\|\xi\|_{W^{1, \infty}\left(\mathbb{R}^{n}\right)}^{2}\|u\|_{W^{1, \infty}(\mathscr{S})}^{2}}{(\log K)^{2}} \sum_{j \in \mathscr{J}} \int_{\eta / K}^{\eta} \frac{r^{n-1}}{r^{2}} d r \\
& \leqslant(1+\delta) \int_{\Omega}|\nabla u(x)|^{2}|\nabla \xi(x)|^{2} d x \\
& \quad+\frac{C_{\delta} C_{\mathscr{S}}\|\xi\|_{W^{1, \infty}\left(\mathbb{R}^{n}\right)}^{2}\|u\|_{W^{1, \infty}(\mathscr{S})}^{2}}{(\log K)^{2}} \int_{\eta / K}^{\eta} \frac{1}{r} d r \\
& =(1+\delta) \int_{\Omega}|\nabla u(x)|^{2}|\nabla \xi(x)|^{2} d x+\frac{C_{\delta} C_{\mathscr{S}}\|\xi\|_{W^{1, \infty}\left(\mathbb{R}^{n}\right)}^{2}\|u\|_{W^{1, \infty}(\mathscr{S})}^{2}}{\log K}
\end{aligned}
$$

This and (1.6) give that

$$
\begin{aligned}
& \int_{\Omega_{\eta, K}}\left(|\nabla u|^{2} \mathscr{K}^{2}+\left|\nabla_{T}\right| \nabla u||^{2}\right) \xi_{\eta, K}^{2} \leqslant \\
& \quad \leqslant(1+\delta) \int_{\Omega}|\nabla u(x)|^{2}|\nabla \xi(x)|^{2} d x+\frac{C_{\delta} C_{\mathscr{S}}\|\xi\|_{W^{1, \infty}\left(\mathbb{R}^{n}\right)}^{2}\|u\|_{W^{1, \infty}(\mathscr{S})}^{2}}{\log K} .
\end{aligned}
$$

We now take $\eta=1 / \log K$ and we send $K \rightarrow+\infty$ (notice that (1.1) allows us to do so), so that we obtain

$$
\int_{\Omega}\left(|\nabla u|^{2} \mathscr{K}^{2}+\left|\nabla_{T}\right| \nabla u||^{2}\right) \xi^{2} \leqslant(1+\delta) \int_{\Omega}|\nabla u|^{2}|\nabla \xi|^{2}
$$

By taking $\delta$ as small as we wish, we obtain (0.3), thus completing the proof of Theorem 1. 


\section{Proof of Theorem 2}

We observe that, under the assumptions of Theorem 2,

$$
u \in W^{1, \infty}(\Omega) \subset C(\bar{\Omega}) .
$$

We suppose, by contradiction, that $\Omega$ is coercive.

Then, $\partial_{n} u>0$, thanks to Theorem 1.3 in [1].

Thus, when $n=2$, the claim of Theorem 2 follows from (0.3) here and Lemma 5.1 in [4].

Thus, we focus on the case in which $n=3$ and $f \geqslant 0$.

For any $t \geqslant 0$ and any $\left(x^{\prime}, x_{3}\right) \in \Omega$, we define

$$
u_{t}\left(x^{\prime}, x_{3}\right):=u\left(x^{\prime}, x_{3}+t\right) .
$$

Due to standard elliptic regularity theory, we have that the following limit exists for any $x^{\prime} \in \mathbb{R}^{2}$, with $\left(x^{\prime}, x_{3}\right) \in \Omega$, and it is attained in $C^{2}\left(\mathbb{R}^{2}\right)$ :

$$
u_{\infty}\left(x^{\prime}\right):=\lim _{t \rightarrow+\infty} u_{t}\left(x^{\prime}, x_{3}\right)
$$

In particular,

$$
\Delta u_{\infty}+f\left(u_{\infty}\right)=0 \quad \text { in } \mathbb{R}^{2} .
$$

We also set

$$
F(r):=\int_{0}^{r} f(s) d s .
$$

Note that $F^{\prime}=f \geqslant 0$ and so $F$ is nondecreasing. Accordingly,

$$
F(u(x)) \leqslant F\left(u_{t}(x)\right) \quad \text { for any } x \in \Omega
$$

and so

$$
F\left(u\left(x^{\prime}, x_{3}\right)\right) \leqslant F\left(u_{\infty}\left(x^{\prime}\right)\right) \quad \text { for any }\left(x^{\prime}, x_{3}\right) \in \Omega .
$$

Now, we take $\Omega_{\epsilon} \subseteq \Omega$ to be a $C^{3}$ coercive epigraph that approaches $\Omega$ when $\epsilon \rightarrow 0^{+}$.

We make use of Lemma 9.1 in [4] (applied here to $u$ in the smooth domain $\Omega_{\epsilon}$ ): we obtain, for any $t \geqslant 0$,

$$
\int_{B_{R} \cap \Omega_{\epsilon}} \frac{|\nabla u|^{2}}{2}-F(u) d x \leqslant C R^{2}+\int_{B_{R} \cap \Omega_{\epsilon}} \frac{\left|\nabla u_{t}\right|^{2}}{2}-F\left(u_{t}\right) d x,
$$

for a suitable constant $C \geqslant 0$. 
Therefore, keeping $t$ fixed and sending $\epsilon \rightarrow 0^{+}$,

$$
\int_{B_{R} \cap \Omega} \frac{|\nabla u|^{2}}{2}-F(u) d x \leqslant C R^{2}+\int_{B_{R} \cap \Omega} \frac{\left|\nabla u_{t}\right|^{2}}{2}-F\left(u_{t}\right) d x .
$$

We now send $t \rightarrow+\infty$ and we conclude that

$$
\int_{B_{R} \cap \Omega} \frac{|\nabla u(x)|^{2}}{2}-F(u(x)) d x \leqslant C R^{2}+\int_{B_{R} \cap \Omega} \frac{\left|\nabla u_{\infty}\left(x^{\prime}\right)\right|^{2}}{2}-F\left(u_{\infty}\left(x^{\prime}\right)\right) d\left(x^{\prime}, x_{3}\right) .
$$

Therefore, from (2.3),

$$
\int_{B_{R} \cap \Omega} \frac{|\nabla u(x)|^{2}}{2} d x \leqslant C R^{2}+\int_{B_{R} \cap \Omega} \frac{\left|\nabla u_{\infty}\left(x^{\prime}\right)\right|^{2}}{2} d\left(x^{\prime}, x_{3}\right) .
$$

We now observe that, by $(2.2)$, it holds that $\Delta u_{\infty} \leqslant 0$ in $\mathbb{R}^{2}$ and therefore, by a classical Liouville Theorem, we have that $u_{\infty}$ is constant.

Hence, (2.4) becomes

$$
\int_{B_{R} \cap \Omega} \frac{|\nabla u(x)|^{2}}{2} d x \leqslant C R^{2}
$$

Thus, in the light of (0.3) and (2.5), we may now apply Corollary 9.4 of [4]: we obtain that $\partial \Omega$ is a hyperplane, in contradiction with the fact that $\Omega$ is coercive.

This completes the proof of Theorem 2 .

\section{References}

[1] Berestycki, H., Caffarelli, L. A. and Nirenberg, L.: Monotonicity for elliptic equations in unbounded Lipschitz domains. Comm. Pure Appl. Math. 50 (1997), no. 11, 1089-1111.

[2] Farina, A.: Propriétés qualitatives de solutions d'équations et systèmes d'équations non-linéaires. Habilitation à diriger des recherches, Paris VI, 2002.

[3] Farina, A., Sciunzi, B. and Valdinoci, E.: Bernstein and De Giorgi type problems: new results via a geometric approach. Ann. Sc. Norm. Super. Pisa Cl. Sci. (5) 7 (2008), no. 4, 741-791.

[4] Farina, A. And Valdinoci, E.: Flattening results for elliptic PDEs in unbounded domains with applications to overdetermined problems. Arch. Ration. Mech. Anal. 195 (2010), no. 3, 1025-1058.

[5] Sternberg, P. And Zumbrun, K.: Connectivity of phase boundaries in strictly convex domains. Arch. Rational Mech. Anal. 141 (1998), no. 4, 375-400. 
[6] Sternberg, P. And Zumbrun, K.: A Poincaré inequality with applications to volume-constrained area-minimizing surfaces. J. Reine Angew. Math. 503 (1998), 63-85.

[7] VÁzquez, J. L.: Personal communication, 2008.

Recibido: 23 de diciembre de 2008

\author{
Alberto Farina \\ LAMFA - CNRS UMR 6140 \\ Université de Picardie Jules Verne \\ Faculté des Sciences \\ 33, rue Saint-Leu \\ 80039 Amiens CEDEX 1, France \\ alberto.farina@u-picardie.fr \\ Enrico Valdinoci \\ Università di Roma Tor Vergata \\ Dipartimento di Matematica \\ via della ricerca scientifica, 1 \\ I-00133 Rome, Italy \\ enrico.valdinoci@uniroma2.it
}

This work has been partially supported by MIUR Variational Methods and Nonlinear Differential Equations and GNAMPA Equazioni nonlineari su varietà: proprietà qualitative e classificazione delle soluzioni. 